\title{
Dietary and weight loss effects on human gut microbiome diversity and metabolism
}

Daniela Fangmann ${ }^{1}$, Femke-Anouska Heinsen², Dominik M. Schulte ${ }^{1}$, Malte-Christoph Rühlemann², Kathrin Türk ${ }^{1}$, Ute Settgast ${ }^{1}$, Nike Müller ${ }^{1}$

Wolfgang Lieb' ${ }^{3}$, John F. Baines ${ }^{4}$, Stefan Schreiber ${ }^{1,2}$, Andre Franke², Matthias Laudes ${ }^{1}$

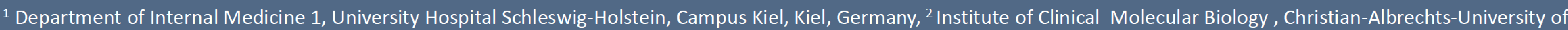
Kiel, Kiel, Germany , ${ }^{3}$ Institute of Epidemiology, Christian-Albrechts-University of Kiel, Kiel, Germany, ${ }^{4}$ Max Planck Institute of Evolutionary Biology, Plön, Germany

\section{INTRODUCTION:}

Recently an association of the gut microbiome and the human energy homeostasis has been shown, suggesting the gut microbiome as a possible target in obesity therapy. Additionally host metabolism is influenced by the gut microbiome, as the transfer of intestinal microbiota from lean donors to type 2 diabetes patients resulted in improved insulin sensitivity of the recipients. Further it has been shown, that nutritional load influences the overall microbial comunity in the gut. Therefore the impact of a multimodal obesity program including a VLCD (approx. $800 \mathrm{kcal} / \mathrm{d}$ ) on gut microbiome an metabolism was examined.

\section{METHODS}

18 obese subjects underwent 3 months VLCD followed by 3 months of weight maintenance. A lean and an obese control group were included. The microbiome was characterized by performing high-throughput dual-indexed 16S rDNA amplicon sequencing.

\begin{tabular}{|c|c|c|c|c|c|c|c|}
\hline & $\begin{array}{l}\text { Dietary Intervention } \\
\text { (VLCD group) }\end{array}$ & $\begin{array}{l}\text { Control Group I } \\
\text { BMI<25 }\end{array}$ & $\begin{array}{c}\text { Control Group II } \\
\text { BMI }>30\end{array}$ & $P_{\text {tootal }}$ & $p_{1}$ & $P_{2}$ & $P_{3}$ \\
\hline gender (\% female) & 83.3 & 84.6 & 84.6 & n.s. & & & \\
\hline age (years) & $47.0(38.8,54.5)$ & $46.0(37.5,50.5)$ & $50.0(38.5,54.0)$ & n.s. & & & \\
\hline height $(m)$ & $1.70(1.61,1.73)$ & $1.68(1.64,1.77)$ & $1.68(1.62,1.73)$ & n.s. & & & \\
\hline weight $(\mathrm{kg})$ & $123.8(114.1,143.5)$ & $64.0(56.7,71.1)$ & $123.5(107.4,138.1)$ & $<0.001$ & $<0.001$ & n.s. & $<0.001$ \\
\hline BMI (kg/m²) & $44.5(38.8,51.5)$ & $22.4(20.7,24.0)$ & $42.3(35.2,47.7)$ & $<0.001$ & $<0.001$ & n.s. & $<0.001$ \\
\hline fasting insulin ( $(\mathrm{UU} / \mathrm{m} \mathrm{l})$ & $15.3(10.4,18.5)$ & $8.6(5.7,10.3)$ & $18.2(12.7,34.8)$ & $<0.001$ & 0.001 & n.s. & $<0.001$ \\
\hline fasting glucose (mg/dli) & $97.0(90.5,114.3)$ & $87.0(83.0,110.0)$ & $106.0(89.0,114.6)$ & n.s. & & & \\
\hline HOMA-IR index & $3.1(2.6,5.1)$ & $1.9(1.3,2.5)$ & $4.8(3.1,12.5)$ & $<0.001$ & 0.001 & n.s. & $<0.001$ \\
\hline \multicolumn{8}{|c|}{ 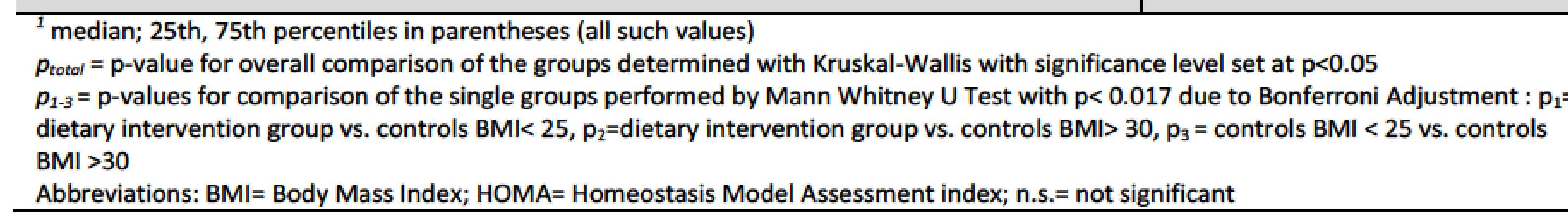 } \\
\hline
\end{tabular}

RESULTS:

Body weight and insulin sensitivity of the intervention group

\begin{tabular}{lccc}
\hline & $\begin{array}{c}\text { O months } \\
\text { (beginning of the study) }\end{array}$ & $\begin{array}{c}\text { 3 months } \\
\text { (end of VLCD) }\end{array}$ & $\begin{array}{c}6 \text { months } \\
\text { (end of weight maintenance) }\end{array}$ \\
\hline weight (kg) & $123.75^{* *}$ & $102.30^{* *}$ & $99.35^{* *}$ \\
& $(114.08,102.25)^{1}$ & $(87.73,120.25)$ & $(87.73-120.05)$ \\
HOMA-IR index & $3.09^{*}$ & $1.73^{*}$ & 2.11 \\
& $(2.61-5.14)$ & $(1.03-3.70)$ & $(1.51-3.87)$ \\
\hline
\end{tabular}

Dingle points of

Abbreviations: HOMA-IR= Homeostasis Model Assessment index

\section{Gut microbiome diversity}

At baseline a significant difference in the Firmicutes/Bacteroidetes ratio between the lean control group and the obese intervention group could be observed $(p=0.047)$

The VLCD resulted in significant alterations in $\beta$-diversity from baseline to 3 months. The changes in diversity diminished during the weight maintenance phase, despite sustained reductions in body weight and sustained improvements of insulin sensitivity.

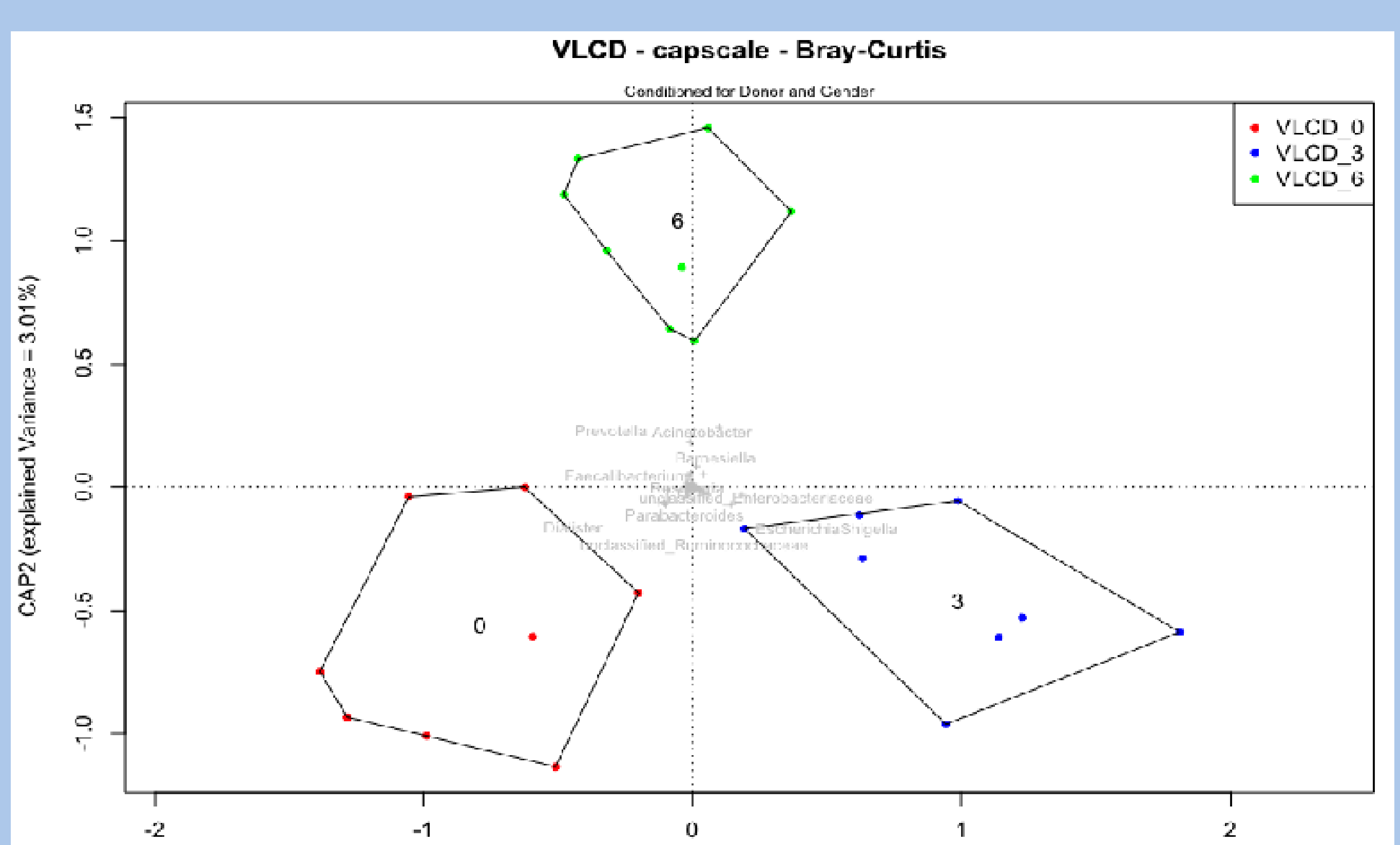

The first coordinate of the CAP explained $6.22 \% \quad(p=0.048)$ of the variance in the microbiota due to the VLCD intervention.

Constrained analysis of principal coordinates (CAP) of the dietary intervention group explaning variance in the microbiota. Baseline $(0$ months $)=0, \quad V L C D$ intervention ( 3 months) $=3$, weight maintenance period $(6$ month $)=6$

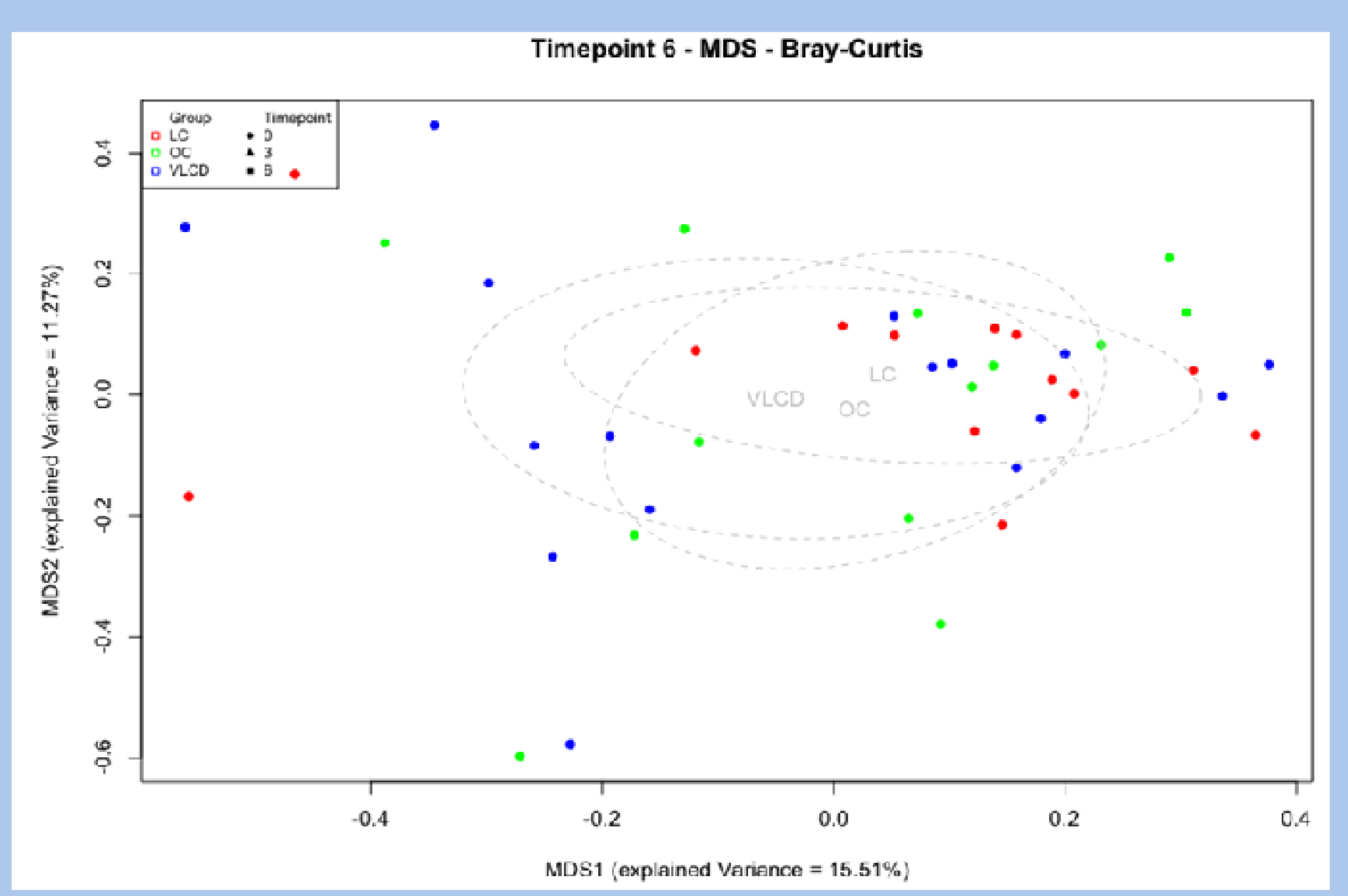

After weight maintenance no significant differences between the three study groups could be observed on phylum or genus level.

Unconstrained Principal Component Analysis

Defined species of the gut microbiome

Acinetobacter represented an indicator species for the observed effect in microbiome diversity (IndVal=0.998; p=0.006).

Bacterial metabolic changes

Metabolic analyses revealed alterations of the bacterial riboflavin pathway from baseline to 3 months (pnom=0.0078). During weight maintenance phase the changes diminished.

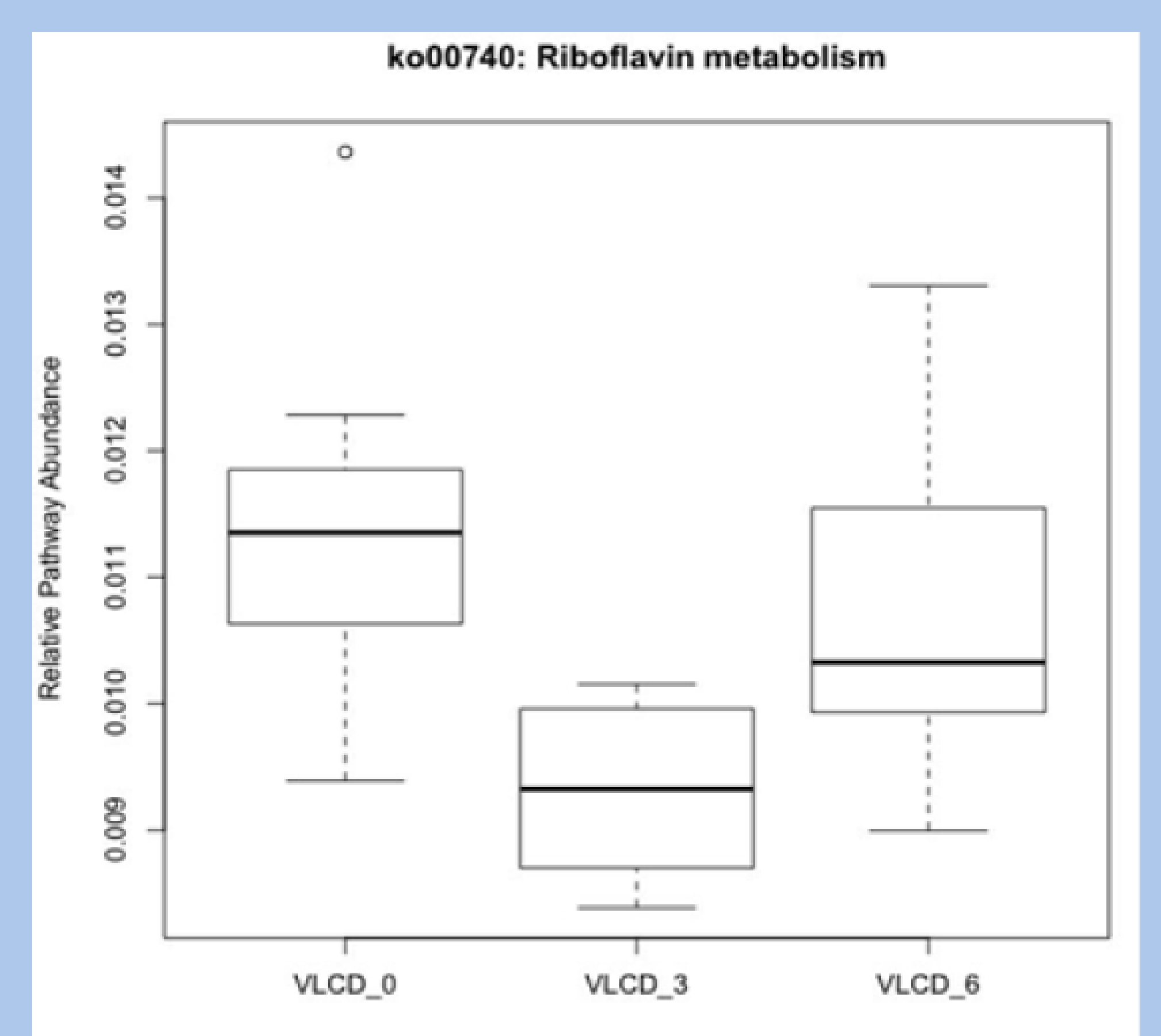

Relative abundance of the riboflavon pathway during the dietary intervention Baseline (0 months) $=0, V L C D$ intervention ( 3 months) $=3$, weight maintenance period ( 6 month) $=6$ nominal statistically significant

\section{CONCLUSION:}

The present data show that in obese humans a VLCD is able to beneficially alter both gut microbiome diversity and metabolism, but also that these changes are not sustained during weight maintenance. This finding might suggest additional measures to target the microbiome during obesity programs. 